\title{
Transition states and the critical parameters of central potentials
}

\author{
Evgeny Z. Liverts and Nir Barnea \\ Racah Institute of Physics, The Hebrew University, Jerusalem 91904, Israel
}

\begin{abstract}
Transition states or quantum states of zero energy appear at the boundary between the discrete part of the spectrum of negative energies and the continuum part of positive energy states. As such, transition states can be regarded as a limiting case of a bound state with vanishing binding energy, emerging for a particular set of critical potential parameters. In this work we study the properties of these critical parameters for short range central potentials. To this end we develop two exact methods and also utilize the first and second order WKB approximations. Using these methods we have calculated the critical parameters for several widely used central potentials.

The general analytic expressions for the asymptotic representations of the critical parameters were derived for cases where either the orbital quantum number $l$ or the number $n$ of bound states approaches infinity.

The above mathematical models enable us to answer the following physical (quantum mechanical) questions: i) what is the number of bound states for a given central potential and given orbital quantum number $l$; ii) what is the maximum value of $l$ which can provide a bound state for the given central potential; iii) what is the order of energy levels for the given form of the central potential.
\end{abstract}

It is revealed that the ordering of energy levels depends on the potential singularity at the origin.

PACS numbers: 03.65.Ge, 03.65.Sq, 03.65.Ta 


\section{INTRODUCTION}

It is generally agreed that estimating the number of bound states of the Schrödinger equation is a problem of great practical importance. A substantial effort was devoted for evaluating the upper and lower limits on the number of bound states for a given central potential. V. Bargmann [1] and J. Schwinger [2] seem to be the first who tackled this problem. Since then many authors have contributed to the study of this problem. Among them we would like to refer to the works [3 7] and to emphasize particularly the contribution of F. Calogero and F. Brau [8 12$]$.

The purpose of the current contribution is to present a systematic approach to the investigation of quantum states with zero energy. These transition states appear between the discrete part of the spectrum of negative energies and the continuum part of positive energies. We study transition states by solving the proper Schrödinger equation for short range central potentials possessing specific sets of critical parameters. To this end, we develop two exact methods for solving the zero energy Schrödinger equation, and for obtaining the values of the associated critical parameters. We apply these methods to find the critical parameters of an improtant class of central potentials including among others the Gaussian and the Yukawa interactions. A few examples of potentials admitting the analytic solutions for the transition states are exhibitted and the associated critical parameters are presented in analytic form. We provide numerical results in the form of tables of the critical parameters. These results enable one to obtain the exact number of bound states for these potentials without any additional computations.

Analyzing our results we have observed the following universal properties of the solutions of the Schrödinger equation with central potentials: (a) For a given orbital angular momentum quantum number $l$ the $n^{\text {th }}$ critical value of the universal parameter $\beta_{n, l}$ of any potential behaves as $n^{2}$ for large $n$. (b) For a given number $n$ of bound states the critical parameter $\beta_{n, l}$ grows as $l^{2}$ with increasing $l$. Both results can be explained with the help of the WKB approximation. (c) The ordering of the energy levels with various $\{n, l\}$ depends on the potential singularity at the origin.

The paper is organized as follows. In Section[I] briefly discuss the Schrödinger equation and the properties of short range potentials. The asymptotic behavior of the solution at the origin and at infinity is discussed in sections III, IV, respectively. In section $\mathrm{V}$ we 
introduce a phase-kind equation for the transition states, and in section VI we describe the WKB approximations for calculation of the critical parameters. The analytic asymptotic expressions for the critical parameters are presented in section VII. Numerical results and conclusions then follow in sections VIII and IX.

\section{SHORT RANGE POTENTIALS}

Let us start this section with a quotation from section $\S 18$ in the Landau \& Lifshitz textbook on quantum mechanics [13]: "If the field diminishes as $-1 / r^{s}$ at infinity, with $s>2$, then there are not levels of arbitrarily small negative energy. The discrete spectrum terminates at a level with a non-zero absolute value, so that the total number of levels is finite".

We notice, that the statement expounded in the first sentence of the above quotation is doubtful. It contradicts our practical experience and also section $\S 133$ of the same book [13]. The conclusion made in the second sentence proves to be true.

Therefore, we shall consider central potentials $V(r)$ satisfying the corresponding boundary condition at infinity:

$$
\lim _{r \rightarrow \infty} r^{2} V(r)=0
$$

Near the origin, the boundary condition for an attractive potential can be written in a similar form

to avoid fall of a particle to the center [13] $(\S \S 18,35)$.

$$
\lim _{r \rightarrow 0} r^{2} V(r)=0
$$

We shall consider the class of potentials which can be presented in the form:

$$
V\left(r, r_{0}\right)=-\frac{g}{r^{s}} f\left(\frac{r}{r_{0}}\right) \quad\left(g>0, r_{0}>0\right),
$$

where $g$ is the coupling constant which determines the strength of the interaction. Note that according to condition (2) the power $s$ must satisfy the inequality

$$
q \equiv s+p<2
$$

where $p$ corresponds to the leading term in series expansion of function $f(r)$ near the origin

$$
f(r) \underset{r \rightarrow 0}{\simeq} \frac{\tilde{g}}{r^{p}} .
$$


It should be realized that the definition (3) describes a wide class of potentials such as, e.g., square well, exponential, Hulthen, Gaussian, Yukawa, Woods-Saxon and many others.

The radial part of the Schrödinger equation for a single particle moving in a central potential field takes the form [13](§32):

$$
\frac{d^{2} \chi}{d r^{2}}+\left\{\frac{2 m}{\hbar^{2}}[E-V(r)]-\frac{l(l+1)}{r^{2}}\right\} \chi=0
$$

where $m$ is the reduced mass, and $\chi \equiv \chi_{l}(r)$ is the reduced radial part of the wave function for a stationary state with angular momentum $l$ and energy $E$.

Changing the potential parameters the appearance of a new bound state is accompanied with a new solution of Eq.(6) for $E=0$. As we limit our discussion to potentials of the form (3), we rewrite Eq.(6) for zero energy in the form

$$
\frac{d^{2} \chi(r)}{d r^{2}}=\left[-\frac{2 m}{\hbar^{2}} \frac{g}{r^{s}} f\left(\frac{r}{r_{0}}\right)+\frac{l(l+1)}{r^{2}}\right] \chi(r) .
$$

The scale transformation $x=r / r_{0}$ leads to the equation:

$$
\frac{d^{2} \tilde{\chi}(x)}{d x^{2}}=\left[-\frac{2 m}{\hbar^{2}} \frac{g r_{0}^{2-s}}{x^{s}} f(x)+\frac{l(l+1)}{x^{2}}\right] \tilde{\chi}(x) .
$$

It is seen that the potentials $V\left(r, r_{0}\right)$ and $r_{0}^{2-s} V(r, 1)$ have equivalent solutions of Eq.(6) . Thus, we are interested in solving the equation

$$
\chi^{\prime \prime}(r)=U(r) \chi(r)
$$

where

$$
\begin{gathered}
U(r)=-\beta v(r)+\frac{l(l+1)}{r^{2}}, \\
v(r)=f(r) r^{-s}
\end{gathered}
$$

Now our choice of the form (3) for central potentials becomes clear. The solutions of Eq.(9) for a given angular momentum quantum number $l$ depend effectively, (see, e.g.,[14]) only on one parameter

$$
\beta=\frac{2 m g}{\hbar^{2}} r_{0}^{2-s}
$$

Eq.(9) is a differential equation of a second order. In order to solve it both analytically and numerically one needs to know the behavior of its solution near the origin and at infinity. 


\section{THE SOLUTION NEAR THE ORIGIN}

At first, let us consider the solution of Eq.(9) near the origin. Expanding the potential into a power series and keeping only the leading term we obtain

$$
\chi^{\prime \prime}(r)=\left[-\frac{\lambda}{r^{q}}+\frac{l(l+1)}{r^{2}}\right] \chi(r)
$$

where

$$
\lambda=\beta \tilde{g}
$$

and $q$ is defined by Eq.(41). The particular solution of Eq.(13), satisfying the boundary condition

$$
\chi(0)=0,
$$

has the form

$$
\chi(r)=A \sqrt{r} J_{\frac{2 l+1}{2-q}}\left(\frac{2 \sqrt{\lambda}}{2-q} r^{1-q / 2}\right),
$$

where $J_{\nu}(z)$ is the Bessel function of the first kind, and $A$ is an arbitrary constant. Keeping the first two terms in the series expansion of the function (16), one obtains

$$
\chi(r) \underset{r \rightarrow 0}{\simeq} A r^{l+1}\left[(2-q)(2 l+3-q)-\lambda r^{2-q}\right] .
$$

In general, for integer and half-integer $q$ the solution of Eq.(9) satisfying the boundary condition (15) can be presented by the following infinite series

$$
\chi(r)=\tilde{A} r^{l+1}\left[1+\sum_{i=1}^{\infty} r^{i}\left(b_{i}+r^{1-q} c_{i}\right)\right] .
$$

For integer $q$ all of the $b$-coefficients should be zero. It follows from Eq.(17) that

$$
c_{1}=-\frac{\lambda}{(2-q)(2 l+3-q)} .
$$

Substituting the representation (18) into Eq.(9), and then equating the expansion coefficients of the same powers of $r$ for the left-hand (lhs) and right-hand sides (rhs) of Eq.(9), one can calculate any finite number of the subsequent coefficients $c_{i}$ and $b_{i}$ with $i \geq 1$.

\section{THE ASYMPTOTIC BEHAVIOR OF TRANSITION STATES}

For an eigenfunction $\Psi$ belonging to the discrete part of the spectrum, the integral $\int|\Psi|^{2} d V$, taken over all space, is finite. This certainly means that $|\Psi|^{2}$ decreases quite 
rapidly, becoming zero at infinity. The motion of the system is limited to a finite range, and it is said to be in a bound state. For wave functions belonging to the continuous part of the spectrum, the integral $\int|\Psi|^{2} d V$ diverges due to the fact that $|\Psi|^{2}$ does not become zero at infinity (or becomes zero insufficiently rapidly).

On the other hand [13] ( $\S 18)$, the spectrum of negative eigenvalues of the energy is discrete, i.e. all states with $E<0$ in the field which vanishes at infinity are bound states. The positive eigenvalues $E>0$, on the other hand, form a continuous spectrum.

In other words, for the bound states the eigenvalues of the energy $E<0$, and the eigenfunctions must satisfy the boundary condition

$$
\lim _{r \rightarrow \infty}|\Psi|^{2}=0
$$

In contrary, for a free state that belongs to the continuous spectrum the energy eigenvalues $E>0$ and $|\Psi|^{2}$ does not become zero at infinity (or becomes zero insufficiently rapidly).

For the transition states with $E=0$ the asymptotic behavior $(r \rightarrow \infty)$ of the eigenfunctions remains unclear.

The important step is to realize that the boundary condition (20) must be valid for the transition states $(E=0)$ as well. Thus, for these states $|\Psi|^{2}$ achieves zero at infinity. However, we note that it may tend to zero too slowly to ensure the convergence of the integral $\int|\Psi|^{2} d V$.

In the following we shall rely on the boundary condition (20) for the transition states.

For $l>0$ the asymptotic boundary condition (1) enables us to neglect the potential $V(r)=-\beta v(r)$ in Eqs.(91)-(11) at large enough $r$. The general solution of the resulting equation has a form

$$
\chi_{l}(r)=C_{1} r^{l+1}+C_{2} r^{-l}
$$

where $C_{1}$ and $C_{2}$ are arbitrary constants. As $\Psi \sim R(r)=\chi_{l}(r) / r$, one should put $C_{1}=0$ in order to satisfy the asymptotic condition (20). Thus, we get

$$
\chi_{l}(r) \underset{r \rightarrow \infty}{\simeq} C_{2} r^{-l}
$$

Expressing the latter equation in the form $r^{l} \chi_{l}(r) \underset{r \rightarrow \infty}{\simeq}$ const, we obtain the following condition for the first derivative:

$$
\lim _{r \rightarrow \infty} \frac{d}{d r}\left[r^{l} \chi_{l}(r)\right] \equiv \lim _{r \rightarrow \infty}\left[l r^{l-1} \chi_{l}(r)+r^{l} \chi_{l}^{\prime}(r)\right]=0
$$


It is clear that the asymptotic behavior of the solution $\chi_{l}(r)$ of Eq.(9) depends on the parameter $\beta$ of the effective potential (10). Thus, according to Eq. (23), the solution $\chi_{l}$ of Eq.(9) fulfills the asymptotic condition

$$
F_{l}\left(\beta_{n}\right) \equiv \lim _{r \rightarrow \infty}\left[\frac{l}{r} \chi_{l}(r)+\chi_{l}^{\prime}(r)\right]=0
$$

for the critical parameters $\beta_{n}$. Here $n$ is a number of zeros of the function $F_{l}(\beta)$ for the given potential (10). Hence, by definition, if for a given $l$ the potential $V(r)$ is characterized by the parameters meeting $\beta_{n+1} \geq \beta>\beta_{n}$ then the proper number of bound states equals $n$.

The asymptotic condition (24) was derived assuming that the orbital quantum number $l>0$. However, it is easy to show that Eq.(24) preserves its validity also for $l=0$. A typical graph of the function $F_{l}(\beta)$ is presented in Fig. 1.

The straightforward solution of the second order differential equation (9) with the boundary conditions (17) and (24) presents our first method for calculating the critical parameters $\beta_{n}$ of a given attractive potential (3) satisfying the boundary conditions (11) and (22). This method is especially effective and accurate for small values of $l$. For a few potentials, such as the exponential, the Hulthen and the Woods-Saxon, one can derive analytical expressions for the critical parameters using this method (see Appendix). However, this is possible only for $S$-states $(l=0)$, when Eq.(9) with the potentials mentioned above has a general analytical solution. We are familiar with only one form of central potential which admits an analytical solution of Eq.(9) for $l \geq 0$. It is a cut-off potential (described in the Appendix) for which the finite square well potential presents its particular case.

Let us add one important comment. We refer to Eq.(24) as the asymptotic behavior condition. However, the solution (21), and therefore - condition (24), correspond to the assumption that the potential $V(r)$ is negligible in comparison with the centrifugal term $l(l+1) / r^{2}$. Therefore, the condition (24) is applicable at a distance $r$ when the condition

$$
|V(r)| \ll \frac{l(l+1)}{r^{2}}
$$

is satisfied.

\section{EQUATION OF THE PHASE KIND}

It was mentioned in the preceding section that the straightforward method for calculating the critical parameters of central potentials loses its accuracy with increasing angular 
momentum quantum number $l$. In this section we propose another method for calculating these parameters. This method is based on the logarithmic derivative $y(r)=\chi^{\prime}(r) / \chi(r)$ of the reduced radial wave function introduced earlier. The final equations are close to, but differ from the so called phase equations presented in [10, 12].

Let us start with the trivial identity

$$
\left(\frac{\chi^{\prime}}{\chi}\right)^{\prime}=\frac{\chi^{\prime \prime}}{\chi}-\left(\frac{\chi^{\prime}}{\chi}\right)^{2}
$$

and transform the radial Schrödinger equation (9) into a Riccati type equation for the corresponding logarithmic derivative $y(r) \equiv y_{l}(r)$ :

$$
y^{\prime}(r)+y^{2}(r)=U(r)
$$

The asymptotic behavior of the logarithmic derivative for $l>0$

$$
y_{l}(r) \underset{r \rightarrow \infty}{\simeq}-\frac{l}{r} \quad(l>0)
$$

follows from the asymptotic representation (22).

To deduce the asymptotic behavior of the logarithmic derivative for the transition $S$ states $(l=0)$ let us start with the fact that for this case the rhs of Eq.(27) equals $V(r)$. Let us then consider central potentials with the asymptotic behavior

$$
V(r) \underset{r \rightarrow \infty}{\simeq}-\frac{\beta}{r^{\mu}} \quad(\beta>0)
$$

where $\mu>2$ according to the boundary condition (11). The general solution of the proper Schrödinger equation (9) has a form:

$$
\chi(r)=\sqrt{r}\left[C_{2} J_{\nu}\left(2 \nu \sqrt{\beta} r^{\frac{1}{2 \nu}}\right)+C_{3} J_{-\nu}\left(2 \nu \sqrt{\beta} r^{\frac{1}{2 \nu}}\right)\right]
$$

with

$$
\nu \equiv \frac{1}{2-\mu}<0 \quad(\mu>2)
$$

It is seen that for $\mu>2$ the argument of the Bessel function goes to zero as $r \rightarrow \infty$. Thus, using series expansion for the Bessel functions, it is easy to show that one should put $C_{2}=0$ in order to satisfy the boundary condition (20). Taking then the logarithmic derivative for the resulting $\chi(r)$, and once more using a series expansion for the Bessel functions, one obtains:

$$
y_{0}(r) \underset{r \rightarrow \infty}{\simeq} \frac{\beta}{\mu-1} r^{1-\mu} .
$$


It is clear that the asymptotic solution (32) of the Riccati equation (27) for $l=0$ satisfies the following inequality:

$$
y_{0}^{2}(r) \underset{r \rightarrow \infty}{\ll}\left|y_{0}^{\prime}(r)\right| .
$$

The asymptotic solution of the Schrödinger equation (9) with the exponential potential is presented in the Appendix (see Eq.(A20) for $l=0$. The corresponding logarithmic derivative

$$
y_{0}^{\exp }(r) \underset{r \rightarrow \infty}{\simeq} \beta e^{-r}
$$

satisfies the inequality (33). It is easy to show that the asymptotic representation $(r \rightarrow \infty)$ of the Hulthen and the Woods-Saxon potentials reduces to the exponential forms $-\beta e^{-r}$ and $-\beta x_{0}^{-1} e^{-r}$, respectively. Hence, the asymptotic solution of the corresponding Eq.(27) can be presented by the rhs of Eq.(34) for the Hulthen potential, and by $\beta x_{0}^{-1} e^{-r}$ for the Woods-Saxon potential. The latter logarithmic derivatives certainly obey the inequality (33) as well.

It is reasonable to suggest that inequality (33) is valid for all of the short range potentials (may be excluding only the cut-off potentials). In this case, one can neglect the square of the logarithmic derivative in the lhs of Eq.(27). The solution of the latter equation with $l=0$ can be obtained then in the explicit form

$$
y_{0}(r) \underset{r \rightarrow \infty}{\simeq} \beta \int_{r}^{\infty} v(r) d r
$$

For potentials with the asymptotic behavior (29), formula (35) gives the asymptotic representation (32). For the exponential potential, the rhs of Eq.(35) leads to (34).

For the Yukawa and Gaussian potentials Eq.(35) yields:

$$
y_{0}^{Y u k}(r) \underset{r \rightarrow \infty}{\simeq} \beta \frac{e^{-r}}{r}, \quad y_{0}^{G a u}(r) \underset{r \rightarrow \infty}{\simeq} \frac{\beta}{2} \frac{e^{-r^{2}}}{r} .
$$

For deriving the latter expressions we used the leading terms of the asymptotic expansions of the incomplete gamma function $\Gamma(0, r)$ and the complementary error function $\operatorname{erfc}(r)$ obtained as the results of integration in Eq.(35).

It is easy to check that the asymptotic logarithmic derivatives (36) satisfy the inequality (33)

The substitution

$$
y_{l}(r)=\mathcal{K}(r) \cot \eta(r)
$$


enables us to transform Eq.(27) into the following equation for the phase function $\eta(r)$ :

$$
\eta^{\prime}(r)=\frac{\mathcal{K}^{\prime}(r)}{2 \mathcal{K}(r)} \sin 2 \eta(r)+\mathcal{K}(r) \cos ^{2} \eta(r)-\frac{U(r)}{\mathcal{K}(r)} \sin ^{2} \eta(r) .
$$

The stabilizing function $\mathcal{K}(r)$ can be chosen in a sufficiently arbitrary manner. The simplest choice is $\mathcal{K}(r)=1$. In this case, the boundary conditions (28) and (35) define the following asymptotic condition for $\eta(r)$ :

$$
\eta(\infty)=-\pi / 2+n \pi . \quad(n=1,2, \ldots)
$$

Any function $\mathcal{K}(r)$ that preserves the limit, $\lim _{r \rightarrow \infty}[y(r) / \mathcal{K}(r)]=0$ provides the asymptotic behavior (39). It was established (at least numerically) that the more precise results are provided by stabilizing functions of the form:

$$
\mathcal{K}_{l}(r)=\sqrt{U_{l}(r)}
$$

where $U_{l}(r)$ governs the behavior of $U(r)$ both near the origin and at infinity, that is

$$
U_{l}(r)=\left\{\begin{array}{ll}
\beta v(r), & l=0 \\
l(l+1) r^{-2}, & l>0
\end{array} .\right.
$$

Use of the stabilizing functions (40)-(41) enables us to replace Eq.(38) with two simpler equations:

$$
\begin{array}{cc}
\eta^{\prime}(r)=\frac{U_{0}^{\prime}(r)}{4 U_{0}(r)} \sin 2 \eta(r)+\sqrt{U_{0}(r)}, & (l=0) \\
\eta^{\prime}(r)=\frac{1}{r}\left[\sqrt{l(l+1)} \cos 2 \eta(r)-\frac{1}{2} \sin 2 \eta(r)\right]+\frac{r U_{0}(r)}{\sqrt{l(l+1)}} \sin ^{2} \eta(r) . & (l>0)
\end{array}
$$

Substituting expressions (40)-(41) for $l>0$ into definition (37) with the asymptotic form (28), one obtains: $\cot \eta(\infty)=-\sqrt{l /(l+1)}$. Thus, the critical parameters $\beta_{n}$ must provide the following asymptotic behavior for the function $\eta(r)$ :

$$
\left.\widetilde{F}_{l}\left(\beta_{n}\right) \equiv \eta(\infty)\right|_{\beta=\beta_{n}}=\delta_{l}-\frac{\pi}{2}+n \pi, \quad(n=1,2, \ldots)
$$

with

$$
\delta_{l}=\arctan \sqrt{\frac{l}{l+1}} .
$$

Eq.(44) was derived for $l>0$. It describes the asymptotic behavior of the solutions of Eq.(43). However, it is clear that the stabilizing function $\mathcal{K}_{0}(r)=\sqrt{U_{0}(r)}$ preserves the 
correctness of the asymptotic formula (39) for the logarithmic derivatives $y_{0}(r)$ with the asymptotic behavior defined by Eq.(35). Therefore, condition (44)-(45) with $l=0$ can be used also for the asymptotic approximation of solutions of Eq.(42).

It is seen from Eq.(42) that the stabilizing function $\mathcal{K}_{0}(r)=\sqrt{U_{0}(r)}$ can be applicable only in case of its nodeless character, otherwise, the simplest choice is $\mathcal{K}_{0}(r)=1$.

The technique described above in this section is sufficient for presentation of the second method for calculating the critical parameters of central potentials of the from (3). However, we would like to make some additional remarks that can be useful.

Eqs.(42), (43), along with the boundary condition (44)-(45), provide a stable and accurate solution to the problem of critical potentials for both small and large values of $l$.

A typical graph of function $t=\widetilde{F}_{l}(\beta)$ has a staircase form. It is presented in Fig, 2 , The abscissas of the points of the staircase function intersections with lines $t=\delta_{l}-\pi / 2+\pi n$ give the desired critical parameters $\beta=\beta_{n}$.

As an additional useful information it can be shown that

$$
\tilde{F}_{l}(0)=\delta_{l}
$$

This is because of the following. Setting $\beta=0$, the second term disappears from the rhs of Eq.(42). The analytic solution to the resultant equation has a form:

$$
\eta(r) \underset{\beta \rightarrow 0}{=} \arctan \left(C \sqrt{U_{0}(r)}\right) .
$$

Condition (11) thus provides $\eta(\infty)=0$ for $\beta=0$ and $l=0$ according to Eq.(47).

Putting $\beta=0$ in Eq.(43), the latter loses the term with $U_{0}(r)$. The resultant equation has analytic solution of the form:

$$
\eta(r) \underset{\beta \rightarrow 0}{=} \arctan \frac{(2 l+1) \tanh \left(\frac{(2 l+1)}{2} \ln r+C\right)-1}{2 \sqrt{l(l+1)}} .
$$

For arbitrary finite real $C$, tanh presented in Eq.(48) approaches 1 as $r \rightarrow \infty$. The resultant expression thus reduces to $\delta_{l}$.

\section{THE FIRST AND SECOND ORDER WKB APPROXIMATIONS}

In this section we apply the first-order and the second-order WKB approaches to calculating the critical parameters of central potentials. Unlike the exact methods presented earlier these methods are certainly approximate but they are also much simpler. 
Specific modification of the first-order WKB approach presented below cannot provide results of high precision. However, its accuracy grows rapidly with increasing the number $n$ of bound states. It is important to note that this method can be applied to transition states with any orbital quantum number $l \geq 0$.

The accuracy of the second-order WKB method grows with increasing $l$. For example, the relative error for $l>10$ can be less than $10^{-5}$. This enables one to test the results obtained by the phase kind method (Sec.V) for large $l$. On the other hand, this method can be used by itself if a very high accuracy is not needed. The disadvantage of this method is its inapplicability for the transition $S$-states $(l=0)$.

For $E=0$ the presence of the centrifugal term in the effective potential $U(r)$ ensures the existence of two turning points for attractive potentials of the form (3). In the second-order approximation [16] the WKB quantization condition [17-19], as applied to our consideration, can be written as

$$
S_{0}+S_{2}=\left(n-\frac{1}{2}\right) \pi, \quad(n=1,2, \ldots)
$$

where $n$ is the number of bound states as in the previous sections. The term

$$
S_{0}=\int_{r_{1}}^{r_{2}} \sqrt{-U(r)} d r
$$

together with the term $S_{1}=-\pi / 2$ correspond to the first order WKB-approximation (for two turning points). The turning points $r_{1}$ and $r_{2}$ are the roots of the equation

$$
\frac{l(l+1)}{r_{i}^{2}}=\beta v\left(r_{i}\right), \quad(i=1,2)
$$

where the function $v(r)$ is defined by Eqs.(3), (11). The second-order correction takes the form [16]

$$
S_{2}=\lim _{\mu \rightarrow+0}\left(\frac{1}{48} \int_{r_{1}+\mu}^{r_{2}-\mu} \frac{U^{\prime \prime}(r)}{[-U(r)]^{3 / 2}} d r-\frac{1}{12 \sqrt{\mu}}\left(b_{1}\left|a_{1}\right|^{-3 / 2}+b_{2}\left|a_{2}\right|^{-3 / 2}\right)\right),
$$

where $a_{1}, b_{1}, a_{2}$ and $b_{2}$ are the expansion coefficients of the effective potential $U(r)$ in the neighborhood of the turning points. That is

$$
U\left(r_{i}+\delta r\right)=a_{i} \delta r+b_{i}(\delta r)^{2}+\ldots
$$

where from Eq.(51) it can be seen that

$$
\begin{aligned}
a_{i} & =-\beta\left[\frac{2}{r_{i}} v\left(r_{i}\right)+v^{\prime}\left(r_{i}\right)\right], \\
b_{i} & =\frac{1}{2} \beta\left[\frac{6}{r_{i}^{2}} v\left(r_{i}\right)-v^{\prime \prime}\left(r_{i}\right)\right] .
\end{aligned}
$$


Notice that the small magnitude of the second-order correction (52) results from the difference of two large terms. Therefore, both these terms must be calculated with high accuracy. Nevertheless, we would like to emphasize that this correction increases the accuracy by two to three orders of magnitude.

A modification of the first-order WKB method, where the centrifugal potential is excluded from the quasiclassical momentum [28], can be applied for calculating the critical parameters under consideration. According to this approach, the quantization condition for the transition states $(E=0)$ reduces to:

$$
\int_{0}^{\infty} \sqrt{\beta v(r)} d r=\pi\left(n+\gamma_{l, q}\right),
$$

where

$$
\gamma_{l, q}=\left\{\begin{array}{lc}
\frac{2 l-1}{4}, & q \leq 0 \\
\frac{2 l-1+q}{2(2-q)}, & 0<q<2
\end{array} .\right.
$$

In accordance to its definition (44), the parameter $q$ is ruled by the behavior of the central potential near the origin. The cut-off potential (A1) presents an exclusion. The exact analytic solution for this case is presented in the Appendix.

\section{THE CRITICAL PARAMETER ASYMPTOTICS}

It was shown in the previous sections that in general the critical parameters for central potentials can be calculated numerically. For a few special cases, presented in the Appendix, one can deduce the analytical results.

In this section it will be shown that it is possible to derive the analytical expressions for the asymptotic form of the critical parameters $\beta \equiv \beta_{n, l}$. In so doing, asymptotic implies a situation where either the number of bound states $n$ approaches infinity for a given finite $l$, or the orbital angular momentum quantum number $l$ goes to infinity for a given finite $n$. The first order WKB approach is applied to solve this problem.

First, let us consider the case of finite $n$ where $l \rightarrow \infty$.

Numerical calculations demonstrate that the distance between turning points $\left|r_{1}-r_{2}\right|$ reaches zero as $l$ approaches infinity. This result can be explained and supported by the following arguments. According to Eqs.(49), (50) the standard Bohr-Sommerfeld quantization 
condition for the transition states reads

$$
\int_{r_{1}}^{r_{2}} \sqrt{-U(r)} d r=\left(n-\frac{1}{2}\right) \pi .
$$

This means that the effective potential $U(r)$ must be negative in the range $\left[r_{1}, r_{2}\right]$. Hence, according to Eq.(10) the critical parameter $\beta$ must tend to infinity as $l^{2}$ or faster for $l \rightarrow \infty$. The latter in turn implies that the integrand in the LHS of Eq.(57) approaches infinity as $l \rightarrow \infty$, whereas the RHS of Eq.(57) remains finite. The above contradiction can be eliminated only by setting $r_{1}=r_{2}$ for the limits of integration, which proves the statement.

It is clear that the point $R_{m}$ of minimum of the effective potential $U(r)$ is localized in the region $\left[r_{1}, r_{2}\right]$, that is

$$
r_{1} \leq R_{m} \leq r_{2}
$$

Therefore, for $l$ approaches infinity $R_{m}$ tends to the point where the turning points $r_{1}$ and $r_{2}$ merge. Hence, in order to calculate $r_{m}=\lim _{l \rightarrow \infty} R_{m}$ it is enough to solve the following set of equations:

$$
\left\{\begin{array}{l}
U\left(r_{m}\right)=0 \\
U^{\prime}\left(r_{m}\right)=0
\end{array}\right.
$$

Substituting the explicit form (10) into (59) and eliminating $\beta$ and $l$, one obtains the following simple equation for $r_{m}$ :

$$
2 v\left(r_{m}\right)+r_{m} v^{\prime}\left(r_{m}\right)=0 .
$$

Now, any of the two equations (59) gives the required asymptotic expression:

$$
\beta_{n, l} \underset{l \rightarrow \infty}{\simeq} \frac{d_{l}}{2} l(l+1)
$$

with

$$
d_{l}=\frac{2}{r_{m}^{2} v\left(r_{m}\right)} .
$$

The second derivatives $d_{l}=d^{2} \beta_{n, l} / d l^{2}$ of the asymptotic critical parameters (61) are presented in Table 1 along with the values of $\Delta_{n, l}=\beta_{n, l+1}-2 \beta_{n, l}+\beta_{n, l-1}$ which approximate the general second derivative $d^{2} \beta_{n, l} / d l^{2}$ numerically. It is seen from this Table that the values of $d_{l}$ are very close to the values of $\Delta_{1,19}$ for the lowest (nodeless) energy states $(n=1)$ and $l=19$. It is worth noting that even though the Yukawa and Gaussian potentials are very different, their asymptotic behaviors are coincident $\left(d_{l}=2 e\right)$. 
TABLE I: The asymptotic representations of critical parameters $\beta_{n, l} \underset{l \rightarrow \infty}{\simeq} \frac{d_{l}}{2} l(l+1)$ and $\beta_{n, l} \underset{n \rightarrow \infty}{\simeq} \frac{d_{n}}{2}\left(n+\gamma_{l, q}\right)^{2}$. The numerical results for $\Delta_{n, l}=\beta_{n, l+1}-2 \beta_{n, l}+\beta_{n, l-1}$ and $\Lambda_{n, l}=\beta_{n+1, l}-2 \beta_{n, l}+\beta_{n-1, l}$ are presented for comparison.

\begin{tabular}{|c|c|c|c|c|c|c|}
\hline potential & exponential & Hulthen & Yukawa & Gaussian & $\mathrm{WS}\left(x_{0}=1\right)$ & $\mathrm{WS}\left(x_{0}=0.001\right)$ \\
\hline \hline$v(r)$ & $e^{-r}$ & $\left(e^{r}-1\right)^{-1}$ & $e^{-r} / r$ & $e^{-r^{2}}$ & $\left(1+x_{0} e^{r}\right)^{-1}$ & $\left(1+x_{0} e^{r}\right)^{-1}$ \\
\hline$r_{m}$ & 2 & 1.59362 & 1 & 1 & 2.21772 & 6.17241 \\
\hline$d_{l}$ & $\frac{e^{2}}{2} / 3.69453 /$ & 3.08828 & $2 e / 5.43656 /$ & $2 e / 5.43656 /$ & 4.14224 & 0.077658 \\
\hline$\Delta_{1,19}$ & 3.69449 & 3.08826 & 5.43654 & 5.43639 & 4.14220 & 0.077664 \\
\hline$\Delta_{5,19}$ & 3.69674 & 3.08845 & 5.43698 & 5.43895 & 4.14471 & 0.077846 \\
\hline $\int_{0}^{\infty} \sqrt{v(r)} d r$ & 2 & $\pi$ & $\sqrt{2 \pi}$ & $\sqrt{\pi / 2}$ & $2 \ln \frac{1+\sqrt{x_{0}+1}}{\sqrt{x_{0}}}$ & $2 \ln \frac{1+\sqrt{x_{0}+1}}{\sqrt{x_{0}}}$ \\
\hline$d_{n}$ & $\frac{\pi^{2}}{2} / 4.93480 /$ & 2 & $\pi / 3.14159 /$ & $4 \pi / 12.5664 /$ & 6.35257 & 0.286909 \\
\hline$\Lambda_{19,0}$ & 4.93480 & 2 & 3.14127 & 12.5694 & 6.35257 & 0.286908 \\
\hline$\Lambda_{19,5}$ & 4.88254 & 1.98432 & 3.11141 & 12.5151 & 6.29585 & 0.286015 \\
\hline$\Lambda_{99,5}$ & 4.930 & 1.998 & 3.138 & 12.561 & 6.347 & 0.2868 \\
\hline
\end{tabular}

Notice that a set of equations (59) for calculating $r_{m}$ cannot be applied to the cut-off potential (A1). The correct result (A24) can be obtained by setting $r_{m}=1$, that is by equating the merging point $r_{m}$ and the matching point $r=1$.

Now let us consider the case of $n \rightarrow \infty$ ( $l$ is finite). To this end, one can successfully employ the modified WKB method [28] presented in the previous section. According to the authors of [28], their method is "exact in the asymptotic limit $n_{r} \rightarrow \infty$ ", where $n_{r}=n-1$. Our numerical results confirm this assertion. Thus, using directly the quantization condition (55), one obtains:

$$
\beta_{n, l} \underset{n \rightarrow \infty}{\simeq} \frac{d_{n}}{2}\left(n+\gamma_{l, q}\right)^{2},
$$

where

$$
d_{n}=2\left(\frac{\pi}{\int_{0}^{\infty} \sqrt{v(r)} d r}\right)^{2},
$$

and the parameter $\gamma_{l, q}$ is defined in Eq.(馬).

For the FSW-like potential Eq.(64) gives $d_{n}=2 \pi^{2}\left(\frac{2-s}{2}\right)^{2}$, which certainly coincides with

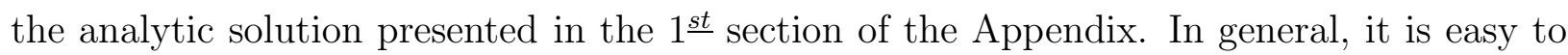


check that the asymptotic expressions for the analytic solutions presented in the Appendix coincide with the results of this section. 


\section{NUMERICAL RESULTS}

The second derivatives $d_{n}=d^{2} \beta_{n, l} / d n^{2}$ of the asymptotic critical parameters (63) are presented in Table \along with values of $\Lambda_{n, l}=\beta_{n+1, l}-2 \beta_{n, l}+\beta_{n-1, l}$ which approximate the general second derivative $d^{2} \beta_{n, l} / d n^{2}$ numerically. It is seen that for the numerical second derivative $\Delta_{n, l}$ the speed $u_{l}$ of a convergence to the asymptotic value $d_{l}(l \rightarrow \infty)$ depends on the number of bound states $n$. The fastest convergence corresponds to the smallest $n=1$. A similar situation is observed for the speed $u_{n}$ of the convergence of $\Lambda_{n, l}$ to $d_{n}(n \rightarrow \infty)$. In this case, the fastest convergence corresponds to the smallest $l=0$. Moreover, Table $\mathbb{I}$ shows that $u_{l}$ far exceeds $u_{n}$.

Using the first and second methods described in Sections [V] and $\mathrm{V}$, respectively, we have computed the critical parameters for a few widely used central potentials included in the nonrelativistic Schrödinger-equation. The results for the exponential, Hulthen, Yukawa and Gaussian potentials are presented in Tables [I, $\mathrm{V}$, respectively. The critical parameters for the Woods-Saxon potential with $x_{0}=1$ and $x_{0}=0.001$ are exhibited in Tables VI and VII, respectively. The first value of parameter $x_{0}$ presents the minimal value of parameter $R=0$. By contrast, the second value of parameter $x_{0}$ corresponds to the case of a large value of $R / r_{0}$ corresponding, e.g., to the optical-model calculations [20].

The critical parameters for the exponential, Hulthen and Woods-Saxon potentials appearing in the Schrödinger equation with the orbital angular momentum quantum number $l=0$ ( $S$-states) can be calculated analytically. For the exponential and Woods-Saxon potentials the requested solutions $(l=0)$ are proportional to the squares of zeros of the corresponding special functions (see Appendix). It is worth noting that the critical parameters for the Hulthen potential $(l=0)$ have an especially simple form. They are equal to $n^{2}$ where the number of bound states equals $n$. All these parameters are displayed in Tables [II, III, VI] and VII for convenient comparing with the cases of $l>0$. Notice that the relative difference between the results obtained by the first method and the analytical ones is less than $10^{-14}$.

The critical parameters for the cut-off potential of the form (A1) are not numerically presented here, because there is no problem to provide the proper calculations according to Eq.(A7) for any orbital quantum number $l$ and parameter $s$. However, we have performed the corresponding computations in order to test both the first and second methods. The relative difference was less than $10^{-12}$ for the second method (Sec.V) for $l \leq 20$. The first 
method provides the same accuracy only for small values of $l \leq 3$, whereas for large $l$ this accuracy can be provided only for $l+n \leq 20$. One should notice that all computations were performed by means of the simplest Mathematica-7 codes using the standard (default) working precision. It is possible, of course, to enhance the calculation accuracy using, e.g., the better working precision.

Due to lack of space we have restricted the results in our Tables to eight significant figures and values of $l+n \leq 16$.

The Yukawa potential is the only one for which we have revealed some earlier results

on critical parameters [21]. They are presented there as the critical screening length for the one-electron eigenstates which were obtained in frame of standard energy calculations. Those results are limited by 5 significant figures and $l+n \leq 9$, and coincide practically with those exhibited here in Table VI.

For the Gaussian potential we have found only the results of the binding energy calculations (see, e.g., Ref.[22-25]). These energies were computed for $l+n \leq 8$ and were completely consistent with the critical parameters presented here in Table VII.

\section{CONCLUSIONS}

In conclusion, we would like to emphasize that the critical parameters are not of some particular character.

First, they present some universal characteristics of central potentials.

Second, using the tables of these parameters, one can answer the following questions:

1) What is the number $n$ of bound states for the given central potential and given orbital quantum number $l$.

2) What is the maximum value of $l$ which can provide a bound state for the given central potential, or vice versa, what is the minimum critical parameter which can provide a bound state with a given $l$ for the given central potential.

3) What is the mutual arrangement (order) of the energy levels $E_{n, l}$ (characterized by the quantum numbers $n$ and $l$ ) for the given form of central potential.

It is clear that the binding energy $E_{n, l}$ rises as the number $n$ of bound states or the orbital angular momentum quantum number $l$ increases. Tables II-VII show that the critical parameters $\beta_{n, l}$ exhibit the same properties in respect to the numbers $\{n, l\}$. It is important 
to realize that for any two sets $\left\{n_{1}, l_{1}\right\}$ and $\left\{n_{2}, l_{2}\right\}$ it follows from inequality

$$
\beta_{n_{1}, l_{1}}>\beta_{n_{2}, l_{2}}
$$

for the given central potential that

$$
E_{n_{1}, l_{1}}>E_{n_{2}, l_{2}}
$$

Therefore, from the presented Tables we can deduce the following important properties of the discrete energy spectrum of the considered central potentials:

$E_{n, l}>E_{n+1, l-1} \quad$ for the Hulthen and Yukawa potentials, $E_{n, l}<E_{n+1, l-1} \quad$ for the exponential, Gaussian and Woods - Saxon potentials.

This probably relates to the fact that the Hulthen and the Yukawa potentials are singular at the origin.

It was established that the leading terms of the asymptotic expansions of the critical parameters $\beta_{n, l}$ have the following forms:

$$
\beta_{n, l} \simeq \begin{cases}a_{l} l^{2} & l \rightarrow \infty \\ a_{n} n^{2} & n \rightarrow \infty\end{cases}
$$

where the general analytic expressions for the factors $a_{l} \equiv d_{l} / 2$ and $a_{n} \equiv d_{n} / 2$ are presented in section VII. The first of the relationships (68) is valid for a finite number $n$ of bound states, whereas the second one is valid for a finite orbital angular momentum quantum number $l$.

\section{ACKNOWLEDGMENTS}

The authors thank Nir Nevo Dinur for useful conversations. 


\section{Appendix A}

\section{Finite square well like potential}

Let us examine potential of the form:

$$
V(r)=\left\{\begin{array}{cl}
-\frac{g}{r^{s}}, & r \leq r_{0} \\
0, & r>r_{0}
\end{array} \quad(s<2)\right.
$$

For $s=0$ one obtains the potential which is widely known as the finite square well. Eq.(9) with the potential (A1) reduces to the form:

$$
\chi^{\prime \prime}(r)=\left[-\frac{\beta}{r^{s}} H(1-r)+\frac{l(l+1)}{r^{2}}\right] \chi(r),
$$

where $H(x)$ is the Heaviside step function. Parameter $\beta$ is defined by Eq.(12). Particular solution satisfying the asymptotic condition (20) and vanishing at the origin has a form:

$$
\chi(r)=\left\{\begin{array}{cc}
A \sqrt{r} J_{\alpha}\left(\frac{\sqrt{\beta}}{\nu} r^{\nu}\right), & r \leq 1 \\
B r^{-l} & r>1
\end{array},\right.
$$

with

$$
\nu=\frac{2-s}{2}, \quad \alpha=\frac{2 l+1}{2-s} .
$$

Here $J_{\alpha}(z)$ is the Bessel function of the first kind, whereas $A$ and $B$ are arbitrary constants. Matching the logarithmic derivatives of solutions (A3) at the point $r=1$, one obtains the following equation for $\beta$ :

$$
\sqrt{\beta}\left[(l+1) J_{\alpha-1}\left(\frac{\sqrt{\beta}}{\nu}\right)-l J_{\alpha+1}\left(\frac{\sqrt{\beta}}{\nu}\right)\right]=-l(2 l+1) J_{\alpha}\left(\frac{\sqrt{\beta}}{\nu}\right) .
$$

Using the properties of the Bessel functions [15], one can reduce Eq. (A5) to the following simplest equation:

$$
J_{\frac{2 l-1+s}{2-s}}\left(\frac{2 \sqrt{\beta}}{2-s}\right)=0
$$

The explicit solution of Eq.(A6) has a form:

$$
\beta_{n}=\left[\left(\frac{2-s}{2}\right) j_{\frac{2 l-1+s}{2-s}, n}\right]^{2}, \quad(n=1,2, \ldots)
$$

where $j_{\mu, n}$ presents the $n^{\text {th }}$ positive zero of the Bessel function $J_{\mu}(z)$. 
From expansion (9.5.12) [15] for large zeros one has:

$$
j_{\nu, n} \simeq\left(n+\frac{\nu}{2}-\frac{1}{4}\right) \pi-\frac{4 \nu^{2}-1}{8\left(n+\frac{\nu}{2}-\frac{1}{4}\right) \pi}-\frac{4\left(4 \nu^{2}-1\right)\left(28 \nu^{2}-31\right)}{192\left(n+\frac{\nu}{2}-\frac{1}{4}\right)^{3} \pi^{3}}-\ldots
$$

Putting

$$
\nu \equiv \nu_{l, s}=\frac{2}{2-s} l-\frac{1-s}{2-s}
$$

and using Eq.(A7), one obtains the following asymptotic expression for the critical parameters corresponding to potential (A1):

$$
\beta_{n} \underset{n \rightarrow \infty}{\simeq}\left(\frac{2-s}{2}\right)^{2} \pi^{2} n^{2}
$$

This yields, in particular:

$$
\frac{d^{2}}{d n^{2}} \beta_{n}(s=0) \underset{n \rightarrow \infty}{\simeq} 2 \pi^{2}, \quad \frac{d^{2}}{d n^{2}} \beta_{n}(s=1) \underset{n \rightarrow \infty}{\simeq} \pi^{2} / 2
$$

From expansion for zeros of the Bessel functions of large order [26] (see, also (9.5.14)[15]), one has:

$$
j_{\nu, n}=\nu\left(1+\sum_{k=1}^{\infty} \alpha_{k, n} \nu^{-\frac{2 k}{3}}\right)
$$

The first coefficients $\alpha_{k, n}$ for $\{k, n\} \leq 5$ can be found in [26]. Thus, using Eq. (A9), one obtains for large enough $l$ :

$$
\beta_{n} \equiv \beta_{n, l}=\left(l-\frac{1-s}{2}\right)^{2}\left[1+\sum_{k=1}^{\infty} \alpha_{k, n}\left(\frac{2 l-1+s}{2-s}\right)^{-\frac{2 k}{3}}\right]^{2}
$$

It is seen that the leading term of the asymptotic $(l \rightarrow \infty)$ expansion of the critical parameter is given by

$$
\beta_{n, l} \underset{l \rightarrow \infty}{\simeq} l^{2}
$$

\section{Exponential potential}

The exponential potential has a form:

$$
V(r)=-g \exp \left(-\frac{r}{r_{0}}\right) .
$$


Eq. (9) with the potential (A15) reads

$$
\chi^{\prime \prime}(r)=\left[-\beta \exp (-r)+\frac{l(l+1)}{r^{2}}\right] \chi(r) .
$$

Parameter $\beta$ is defined here by Eq.(12) with $s=0$. Eq.(A16) has an analytical solution only for the case of $l=0$. Such a particular solution satisfying the boundary condition (15) has a form:

$$
\chi(r)=A\left[J_{0}(q) Y_{0}\left(q e^{-\frac{r}{2}}\right)-Y_{0}(q) J_{0}\left(q e^{-\frac{r}{2}}\right)\right],
$$

where $q=2 \sqrt{\beta}$, whereas $J_{0}(z)$ and $Y_{0}(z)$ are the Bessel functions of the first and second kind, respectively. The argument of the Bessel functions in Eq.(A17) achieves zero as $r \rightarrow \infty$. Therefore, using series expansion for the Bessel functions [15]

$$
J_{0}\left(q e^{-\frac{r}{2}}\right) \underset{r \rightarrow \infty}{\simeq} 1-\frac{q^{2}}{4} e^{-r}, \quad Y_{0}\left(q e^{-\frac{r}{2}}\right) \underset{r \rightarrow \infty}{\simeq}-\frac{r}{\pi}
$$

one should put

$$
J_{0}(q)=0
$$

in order solution (A17) satisfies the asymptotic boundary condition (20). Taking into account condition (A19) for the transition state, and the first of expansions (A18) one can write for the asymptotic behavior of the solution (A17):

$$
\chi(r) \underset{r \rightarrow \infty}{\simeq}-A Y_{0}(2 \sqrt{\beta})\left(1-\beta e^{-r}\right) .
$$

From Eq. (A19) one obtains for the critical parameters:

$$
\beta_{n}=\frac{1}{4} j_{0, n}^{2}, \quad(n=1,2, \ldots)
$$

where $j_{0, n}$ presents the $n^{t h}$ positive zero of the Bessel function $J_{0}(z)$.

Putting $\nu=0$ in expansion (A8) for large zeros, one obtains:

$$
j_{0, n} \simeq\left(n-\frac{1}{4}\right) \pi+\frac{1}{2(4 n-1) \pi}-\frac{124}{3(4 n-1)^{3} \pi^{3}}+\ldots
$$

This yields

$$
\beta_{n} \underset{n \rightarrow \infty}{\simeq} \frac{\pi^{2}}{4}\left(n-\frac{1}{4}\right)^{2} .
$$

The value of second derivative $\lim _{n \rightarrow \infty} d^{2} \beta_{n} / d n^{2}=\pi^{2} / 2$ coincides with the corresponding value (A11) for the FSW-like potential with $s=1$. 


\section{Hulthen potential}

For the Hulthen potential

$$
V(r)=-\frac{g e^{-\frac{r}{r_{0}}}}{1-e^{-\frac{r}{r_{0}}}},
$$

Eq.(9) becomes

$$
\chi^{\prime \prime}(r)=\left[-\frac{\beta}{e^{r}-1}+\frac{l(l+1)}{r^{2}}\right] \chi(r) .
$$

For this differential equation one can obtain a general analytic solution of the form

$$
\chi(r) \equiv \phi(x)=A x_{2}^{-\alpha} F_{1}(-\alpha,-\alpha ; 1-2 \alpha ; x)+B x_{2}^{\alpha} F_{1}(\alpha, \alpha ; 1+2 \alpha ; x)
$$

with

$$
\alpha=\sqrt{\beta}, \quad x=e^{r},
$$

only for $S$-states $(l=0)$. Here ${ }_{2} F_{1}(a, b ; c ; z)$ is the Gauss hypergeometric function, $A$ and $B$ are arbitrary constants.

Formulas (15.1.20) and (6.1.18) [15] yield:

$$
{ }_{2} F_{1}(a, a ; 1+2 a ; 1)=\frac{4^{a} \Gamma(a+1 / 2)}{\sqrt{\pi} \Gamma(a+1)},
$$

where $\Gamma(z)$ denotes Euler's gamma function. The latter representation enables us to obtain the vanishing at the origin $(r \rightarrow 0 \Rightarrow x \rightarrow 1)$ solution in the form:

$$
\begin{aligned}
\chi(r) \equiv \varphi(x)=C\left[\left(\frac{4}{x}\right)^{\alpha} \Gamma(1-\alpha) \Gamma\left(\frac{1}{2}+\alpha\right){ }_{2} F_{1}(-\alpha,-\alpha ; 1-2 \alpha ; x)-\right. \\
\left.\left(\frac{x}{4}\right)^{\alpha} \Gamma(1+\alpha) \Gamma\left(\frac{1}{2}-\alpha\right){ }_{2} F_{1}(\alpha, \alpha ; 1+2 \alpha ; x)\right],
\end{aligned}
$$

with arbitrary constant $C$. For examination of the asymptotic behavior of solution (A29), one can use formula (15.3.13) [15], which yields:

$$
(-x)^{a}{ }_{2} F_{1}(a, a ; 1+2 a ; x) \underset{x \rightarrow \infty}{\simeq} \frac{2 \Gamma(2 a)}{\Gamma^{2}(a)} \ln (-x) .
$$

Inserting the asymptotic representation (A30) for $a=\alpha$ and $a=-\alpha$ into the rhs of Eq.(A29), one obtains after some transformations:

$$
\chi(r) \underset{r \rightarrow \infty}{\simeq}-2 C \alpha \sqrt{\pi}(i \pi+r) .
$$

On the other hand, series expansion of solution (A29) near $x=1(r \rightarrow 0)$ yields:

$$
\chi(r) \underset{r \rightarrow 0}{\simeq}-2 C \alpha^{2} \pi^{3 / 2} \csc (\pi \alpha) r .
$$


Putting

$$
C=-\left[2 \alpha^{2} \pi^{3 / 2} \csc (\pi \alpha)\right]^{-1}
$$

one can get rid of $\alpha$-dependence for the leading term of the $\chi(r)$ series expansion. Substituting expression (A33) into the asymptotic representation (A31), one finally obtains:

$$
\chi(r) \underset{r \rightarrow \infty}{\simeq} \frac{\sin (\pi \alpha)}{\pi \alpha}(i \pi+r) .
$$

Thus, in order to satisfy asymptotic condition (20), one should put:

$$
\sin (\pi \alpha)=0 . \quad(\alpha \neq 0)
$$

The roots of Eq. A35) are the integers, that is $\alpha_{n}=n$. Thus, from definition (A27) one obtains that the critical parameters for the Hulthen potential can be determined from the simplest relation:

$$
\frac{2 m g r_{0}^{2}}{\hbar^{2}}=n^{2}, \quad(n=1,2, \ldots)
$$

where $n$ is a number of $S$-bound states.

\section{Woods-Saxon potential}

Finally, let us examine the Woods-Saxon potential

$$
V(r)=-\frac{g}{1+\exp \left(\frac{r-R}{r_{0}}\right)} \quad(R>0)
$$

which is the most complicated one. For this case Eq.(9) takes a form:

$$
\chi^{\prime \prime}(r)=\left[-\frac{\beta}{1+x_{0} e^{r}}+\frac{l(l+1)}{r^{2}}\right] \chi(r),
$$

where $\beta$ is defined by Eq.(12) with $s=0$, whereas $x_{0}=\exp \left(-R / r_{0}\right)$. Eq.(A38) admits analytical solution only for the case of $l=0$. Introducing a new variable $x=x_{0} e^{r}$ and a new parameter $\alpha=\sqrt{\beta}$, one obtains a new differential equation

$$
x^{2} \varphi^{\prime \prime}(x)+x \varphi^{\prime}(x)+\frac{\alpha^{2}}{1+x} \varphi(x)=0
$$

for function $\varphi(x) \equiv \chi(r)$. The vanishing at the origin solution of Eq. (A39) has a form:

$$
\varphi(x)=C\left[F\left(-\alpha, x_{0}\right) F(\alpha, x)-F\left(\alpha, x_{0}\right) F(-\alpha, x)\right]
$$


where

$$
F(\alpha, x)=x_{2}^{i \alpha} F_{1}(i \alpha, i \alpha ; 1+2 i \alpha ;-x)
$$

Here ${ }_{2} F_{1}(a, b ; c ; z)$ is the Gauss hypergeometric function, $C$ is arbitrary constant. For considered case, formula (15.3.13) [15] yields

$$
(x)^{b}{ }_{2} F_{1}(b, b ; 1+2 b ;-x) \underset{x \rightarrow \infty}{\simeq} \frac{2 \Gamma(2 b)}{\Gamma^{2}(b)} \ln (x) .
$$

Inserting the latter representation into solution (A40) and returning to the initial variable $r$, one obtains:

$$
\begin{array}{r}
\chi(r) \underset{r \rightarrow \infty}{\simeq} 2 C\left(r+\ln x_{0}\right)\left[\frac{F\left(-\alpha, x_{0}\right) \Gamma(2 i \alpha)}{\Gamma^{2}(i \alpha)}-\frac{F\left(\alpha, x_{0}\right) \Gamma(-2 i \alpha)}{\Gamma^{2}(-i \alpha)}\right]= \\
2 C\left(r+\ln x_{0}\right) i \alpha{ }_{2} F_{1}\left(-i \alpha, i \alpha ; 1 ;-\frac{1}{x_{0}}\right) .
\end{array}
$$

Thus, to satisfy the condition (20) for the transitional states, one should put

$$
{ }_{2} F_{1}\left(-i \alpha, i \alpha ; 1 ;-\frac{1}{x_{0}}\right)=0 .
$$

The roots $\beta_{n}=\alpha_{n}^{2}$ of the latter transcendental equation present the desired critical parameters for a given $x_{0}$. It is worth noting that Eq.(A44) can be simplified if one uses the following relationships between the Gauss hypergeometric functions, the Jacobi functions $P_{\nu}^{(a, b)}$ and the Legendre functions $P_{\nu}$ :

$$
\begin{array}{r}
{ }_{2} F_{1}(-i \alpha, i \alpha ; 1 ;-z)=P_{i \alpha}^{(0,-1)}(2 z+1)=(1+z) P_{i \alpha-1}^{(0,1)}(2 z+1)=\operatorname{Re}\left[P_{i \alpha}(2 z+1)\right] \\
(\alpha>0, z>0) .
\end{array}
$$

According to the asymptotic expansion for the Legendre function of imaginary degree (see, Eq.(3.2) [27]), one has

$$
R e\left[P_{i \alpha}(\cosh t)\right] \simeq \frac{1}{\sqrt{2}} \sum_{k=0}^{N}(2 k-1) ! ! a_{k}(t)\left(-\frac{t}{\alpha}\right)^{k} J_{k}(\alpha t)+O\left(\alpha^{-N-1}\right)
$$

where

$$
a_{0}(t)=\sqrt{t \operatorname{coth}\left(\frac{t}{2}\right)}, \quad a_{1}(t)=\frac{a_{0}(t)}{8 t}\left(\frac{\cosh t-2}{\sinh t}+\frac{1}{t}\right) .
$$

In zero approximation for large enough $\alpha$, one can put $N=0$, whence

$$
R e\left[P_{i \alpha}(\cosh t)\right] \underset{\alpha \rightarrow \infty}{\simeq} \frac{1}{\sqrt{2}} \sqrt{t \operatorname{coth}\left(\frac{t}{2}\right)} J_{0}(\alpha t)
$$


Thus, roots of Eq. (A44) for large enough $\alpha=\sqrt{\beta}$ are very close to zeros of the Bessel function $J_{0}(\alpha t)$, where

$$
t=\operatorname{arccosh}\left(\frac{2}{x_{0}}+1\right)=2 \operatorname{arcsinh}\left(\frac{1}{\sqrt{x_{0}}}\right)=2 \ln \left(\frac{1+\sqrt{x_{0}+1}}{\sqrt{x_{0}}}\right) .
$$

In terms of zeros $j_{0, n}$ of the Bessel functions, one can then write down:

$$
j_{0, n} \simeq 2 \sqrt{\beta} \ln \left(\frac{1+\sqrt{x_{0}+1}}{\sqrt{x_{0}}}\right) .
$$

Taking into account expansion (A22), one obtains finally:

$$
\beta_{n} \underset{n \rightarrow \infty}{\simeq} \frac{\pi^{2}}{4 \ln ^{2}\left(\frac{1+\sqrt{x_{0}+1}}{\sqrt{x_{0}}}\right)}\left(n-\frac{1}{4}\right)^{2} .
$$

[1] V. Bargmann, Proc. Natl. Acad. Sci. USA 38, 961 (1952).

[2] J. Schwinger, Proc. Natl. Acad. Sci. USA 47, 122 (1961).

[3] M. F. Barnsley, J. Phys. A: Math.Gen. 11, 55 (1978).

[4] R. Subramanian and K. V. Bhagwat, J. Phys. A: Math.Gen. 20, 69 (1987).

[5] F. M. Fernandez, J. F. Ogilvie, and R. H. Tipping, Phys. Lett. 116, 407 (1986).

[6] F. M. Fernandez, Int. J. Quant. Chem. 25, 95 (1991).

[7] Dai Shi, J. Math. Phys. 51, 083523 (2010).

[8] F. Calogero, Commun. math. Phys. 1, 80 (1965).

[9] F. Brau, Phys. Rev. A 70, 062112 (2004); J. Phys. A: Math. Gen. 37, 6687 (2004).

[10] F. Brau and F. Calogero, J. Phys. A: Math. Gen. 36, 12021 (2003); J. Math. Phys. 44, 1554 (2003).

[11] F. Brau and F. Calogero, Phys. Lett. A 321, 225 (2004).

[12] F. Calogero, Variable phase approach to potential scattering (New York and London: Acad. Press, 1967)

[13] L. D. Landau and E. M. Lifshitz, Quantum Mechanics. Non-relativistic theory (Oxford, New York,..., Toronto: Pergamon Press, 1977, 3-rd edition)

[14] S. H. Patil, Phys. Rev. A 24, 3038 (1981).

[15] M. Abramowitz and I. Stegun, Handbook of Mathematical Functions with Formulas, Graphs, and Mathematical Tables (Moscow: Nauka, 1979) 
[16] T. Nishigori, Chem. Phys. Lett. 221, 492 (1994).

[17] C. M. Bender, K. Olaussen, and P. S. Wang, Phys. Rev. D 16, 1740 (1977).

[18] C. M. Bender and S. A. Orszag, Advanced Mathematical Methods for Scientists and Engineers (New York, Berlin,..: Springer, 1999)

[19] R. Adhikari, R. Dutt, A. Khare, and U. P. Sukhatme, Phys. Rev. A 38, 1679 (1988).

[20] J. P. Jeukenne, A. Lejeune, and C. Mahaux, Phys. Rev. C 10, 1391 (1974); M. Jaminon, J. P. Jeukenne, and C. Mahaux, Phys. Rev. C 34, 468 (1986).

[21] F. J. Rogers, H. C. Graboske Jr. and D. J. Harwood, Phys. Rev A 1, 1577 (1970).

[22] A. Chatterjee, J. Phys. A: Math. Gen. 18, 2403 (1985).

[23] C. S. Lai, J. Phys. A: Math. Gen. 16, L181 (1983).

[24] R. E. Crandall, J. Phys. A: Math. Gen. 16, L395 (1983).

[25] N. Bessis, G. Bessis and B. Joulakian, J. Phys. A: Math. Gen. 15, 3679 (1982).

[26] F. W. J. Olver, Proc. Cambridge Philos. Soc. 47, 699 (1951).

[27] P. Malits, J. Math. Anal. Appl. 281, 205 (2003).

[28] B. M. Karnakov, V. D. Mur, and V. S. Popov, J. Exp. Theor. Phys. 89, 271 (1999). 
TABLE II: Critical parameters $\beta=2 m g r_{0}^{2} \hbar^{-2}$ of the exponential potential $V(r)=-g \exp \left(-r / r_{0}\right)$.

\begin{tabular}{|c|c|c|c|c|c|c|c|c|c|}
\hline$n \backslash l$ & 0 & 1 & 2 & 3 & 4 & 5 & 6 & 7 & 8 \\
\hline 1 & 1.4457965 & 7.0490613 & 16.312928 & 29.258323 & 45.892427 & 66.218077 & \multicolumn{3}{|c|}{90.236557117 .94852149 .35431} \\
\hline 2 & 7.6178156 & 16.921126 & 29.879667 & 46.518231 & 66.845206 & 90.863808 & \multicolumn{3}{|c|}{118.57542149 .98069185 .07997} \\
\hline 3 & 18.721752 & 31.525958 & 48.076670 & 68.345880 & 92.323606 & 120.00486 & \multicolumn{3}{|c|}{151.38676186 .46751225 .24597} \\
\hline 4 & 34.760071 & 50.947660 & 71.002111 & 94.837734 & 122.41832 & 153.72542 & \multicolumn{3}{|c|}{188.74853227 .48127269 .91954} \\
\hline 5 & 55.733076 & 75.226301 & 98.713352 & 126.05709 & 157.19351 & 192.08825 & \multicolumn{3}{|c|}{230.72116273 .07970319 .15566} \\
\hline 6 & 81.640838 & 104.38402 & 131.24653 & 162.04738 & 196.69590 & 235.14112 & \multicolumn{3}{|c|}{277.35218323 .30940372 .99967} \\
\hline 7 & 112.48338 & 138.43438 & 168.62580 & 202.83958 & 240.96044 & 282.92106 & \multicolumn{3}{|c|}{328.67953378 .20847431 .48931} \\
\hline 8 & 148.26072 & 177.38629 & 210.86809 & 248.45649 & 290.01388 & 335.45733 & \multicolumn{3}{|c|}{384.73391437 .80834494 .65627} \\
\hline 9 & 188.97285 & 221.24597 & 257.98574 & 298.91536 & 343.87710 & 392.77335 & \multicolumn{2}{|c|}{445.54045502 .13521} & \\
\hline 10 & 234.61978 & 270.01793 & 309.98801 & 354.22957 & 402.56672 & 454.88817 & 511.11996 & 488.5090 & \\
\hline 11 & 285.20151 & 323.70558 & 366.88206 & 414.40969 & 466.09616 & 521.81745 & 483.54571 & 426.4139 & 6 \\
\hline 12 & 340.71804 & 382.31153 & 428.67350 & 479.46420 & 534.47640 & 479.64012 & 422.43924 & 368.94348 & 5 \\
\hline 13 & 401.16937 & 445.83785 & 495.36688 & 549.40007 & 476.68376 & 419.44387 & 365.90248 & 316.06058 & 4 \\
\hline 14 & 466.55550 & 514.28624 & 566.96589 & 474.58293 & 417.32411 & 363.76075 & 313.89306 & 267.72133 & 3 \\
\hline 15 & 536.87643 & 587.65804 & 473.25639 & 415.99092 & 362.41992 & 312.54336 & 266.36122 & 223.87344 & 2 \\
\hline 16 & 612.13217 & 472.63339 & 415.36754 & 361.79612 & 311.91912 & 265.736481 & 223.24818 & 184.45415 & 1 \\
\hline & & 15 & 14 & 13 & 12 & 11 & 10 & 9 & $l \backslash n$ \\
\hline
\end{tabular}


TABLE III: Critical parameters $\beta=2 m g r_{0}^{2} \hbar^{-2}$ of the Hulthen potential $V(r)=-g \exp \left(-r / r_{0}\right) /\left[1-\exp \left(-r / r_{0}\right)\right]$.

\begin{tabular}{|c|c|c|c|c|c|c|c|c|c|}
\hline$n \backslash l$ & 0 & 1 & 2 & 3 & 4 & 5 & 6 & 7 & 8 \\
\hline 1 & 1 & 5.3059406 & 12.685368 & 23.146783 & 36.693671 & 53.327421 & 73.048651 & 95.857670 & 121.75465 \\
\hline 2 & 4 & 10.724673 & 20.499398 & 33.348492 & 49.279726 & 68.296123 & 90.399036 & 115.58915 & 143.86682 \\
\hline 3 & 9 & 18.100968 & 30.253614 & 45.480804 & 63.790287 & 85.185101 & 109.66659 & 137.23540 & 167.89189 \\
\hline 4 & 16 & 27.448626 & 41.962702 & 59.557425 & 80.237830 & 104.00568 & 130.86162 & 160.80588 & 193.83855 \\
\hline 5 & 25 & 38.775435 & 55.636363 & 75.588244 & 98.631880 & 124.76686 & 153.99258 & 186.30851 & 221.71423 \\
\hline 6 & 36 & 52.086245 & 71.281353 & 93.580632 & 118.97988 & 147.47591 & 179.06647 & 213.74998 & 251.52532 \\
\hline 7 & 49 & 67.384298 & 88.902568 & 113.54024 & 141.28775 & 172.13879 & 206.08916 & 243.13600 & 283.27733 \\
\hline 8 & 64 & 84.671878 & 108.50368 & 135.47149 & 165.56029 & 198.76046 & 235.06562 & 274.47149 & 316.97510 \\
\hline 9 & 81 & 103.95066 & 130.08753 & 159.37792 & 191.80146 & 227.34506 & 266.00010 & 307.76072 & \\
\hline 10 & 100 & 125.22192 & 153.65633 & 185.26240 & 220.01452 & 257.89613 & 298.89626 & 326.51175 & 7 \\
\hline 11 & 121 & 148.48665 & 179.21189 & 213.12731 & 250.20224 & 290.41668 & 336.34850 & 292.39168 & 6 \\
\hline 12 & 144 & 173.74563 & 206.75567 & 242.97462 & 282.36696 & 346.46731 & 301.79385 & 260.20943 & 5 \\
\hline 13 & 169 & 200.99951 & 236.28888 & 274.80601 & 356.85339 & 311.46707 & 269.16916 & 229.95964 & 4 \\
\hline 14 & 196 & 230.24882 & 267.81254 & 367.49439 & 321.39765 & 278.38907 & 238.46863 & 201.63626 & 3 \\
\hline 15 & 225 & 261.49399 & 378.38000 & 331.57422 & 287.85660 & 247.22711 & 209.68569 & 175.23229 & 2 \\
\hline 16 & 256 & 389.50148 & 341.98731 & 297.56137 & 256.22366 & 217.97415 & 182.81284 & 150.73968 & 1 \\
\hline & & 15 & 14 & 13 & 12 & 11 & 10 & 9 & $l \backslash n$ \\
\hline
\end{tabular}


TABLE IV: Critical parameters $\beta=2 m g r_{0} \hbar^{-2}$ of the Yukawa potential $V(r)=-g \exp \left(-r / r_{0}\right) / r$.

\begin{tabular}{|c|c|c|c|c|c|c|c|c|}
\hline$n \backslash l$ & 0 & 1 & 2 & 3 & 4 & 5 & 7 & 8 \\
\hline 1 & 1.6798078 & 9.0819590 & 21.894984 & 40.135552 & 63.808976 & 92.917164127 .46092 & 167.44064 & 212.85654 \\
\hline 2 & 6.4472603 & 17.744576 & 34.420414 & 56.514114 & 84.036777 & 116.99234155 .38248 & 199.20797 & 248.46926 \\
\hline 3 & 14.342028 & 29.461426 & 49.969576 & 75.899394 & 107.26037 & 144.05569186 .28656 & 233.95349 & 287.05673 \\
\hline 4 & 25.371660 & 44.261254 & 68.571467 & 98.317925 & 133.50366 & 174.12875220 .19272 & 271.69505 & 328.63535 \\
\hline 5 & 39.538842 & 62.160193 & 90.245270 & 123.78892 & 162.78498 & 207.22876257 .11705 & 312.44769 & 373.21919 \\
\hline 6 & 56.84486 & 83.168247 & 115.00434 & 152.32675 & 195.11871 & 243.36968297 .07295 & 356.22417 & 420.82039 \\
\hline 7 & 77.290455 & 107.29208 & 142.85836 & 183.94242 & 230.51632 & 282.56299340 .07168 & 403.03541 & 471.44949 \\
\hline 8 & 100.87607 & 134.53636 & 173.81459 & 218.64457 & 268.98711 & 324.81824386 .12280 & 452.89086 & 525.11571 \\
\hline 9 & 127.60202 & 164.90453 & 207.87862 & 256.44012 & 310.53874 & 370.14347435 .23449 & 505.79870 & \\
\hline 10 & 157.46853 & 198.39917 & 245.05485 & 297.33466 & 355.17758 & 418.54550487 .41381 & 545.31066 & 7 \\
\hline 11 & 190.47575 & 235.02231 & 285.34681 & 341.33284 & 402.90900 & $\begin{array}{l}470.03018 \\
566.34038\end{array}$ & 490.8596 & 6 \\
\hline 12 & 226.62381 & 274.77554 & 328.75740 & 388.43851 & 453.73757 & 588.17003511 .08099 & 439.43054 & 5 \\
\hline 13 & 265.91281 & 317.66016 & 375.28899 & 438.65494 & 610.77114 & 532.08136458 .82871 & 391.01331 & 4 \\
\hline 14 & 308.34282 & 363.67724 & 424.94360 & 634.12021 & 553.83444 & 478.98522409 .57254 & $=345.59639$ & 3 \\
\hline 15 & 353.91391 & 412.82768 & 658.19765 & 576.31864 & 499.87607 & 428.86991363 .30010 & 303.16658 & 2 \\
\hline 16 & 402.62614 & 682.98698 & 599.51605 & 521.48163 & 448.88371 & 381.72227319 .99729 & 263.70873 & 1 \\
\hline & & 15 & 14 & 13 & 12 & 11 & 9 & $l \backslash n$ \\
\hline
\end{tabular}


TABLE V: Critical parameters $\beta=2 m g r_{0}^{2} \hbar^{-2}$ of the Gaussian potential $V(r)=-g \exp \left(-r^{2} / r_{0}^{2}\right)$.

\begin{tabular}{|c|c|c|c|c|c|c|c|c|c|}
\hline$n \backslash l$ & 0 & 1 & 2 & 3 & 4 & 5 & 6 & 7 & 8 \\
\hline 1 & 2.6840047 & 12.099309 & 26.901078 & 47.107862 & 72.733004 & 103.78395 & 140.26479 & 182.17789 & 229.52463 \\
\hline 2 & 17.795700 & 35.089777 & 57.675772 & 85.627393 & 118.97711 & 157.74107 & 201.92788 & 251.54239 & 306.58749 \\
\hline 3 & 45.573480 & 70.482856 & 100.71505 & 136.32500 & 177.33962 & 223.77276 & 275.63189 & 332.92115 & 395.64293 \\
\hline 4 & 85.963400 & 118.35165 & 156.12749 & 199.31846 & 247.93811 & 301.99288 & 361.48565 & 426.41761 & 496.78909 \\
\hline 5 & 138.94811 & 178.72771 & 223.97020 & 274.67746 & 330.84800 & 392.47880 & 459.56651 & 532.10794 & 610.10035 \\
\hline 6 & 204.51926 & 251.62783 & 304.27712 & 362.44675 & 426.12056 & 495.28556 & 569.93140 & 650.04986 & 735.63442 \\
\hline 7 & 282.67201 & 337.06209 & 397.07024 & 462.65690 & 533.79230 & 610.45368 & 692.62349 & 780.28811 & 873.43695 \\
\hline 8 & 373.40327 & 435.03714 & 502.36469 & 575.32978 & 653.8902 & 738.01395 & 827.67628 & 922.85815 & 1023.5447 \\
\hline 9 & 476.71088 & 545.55758 & 620.17137 & 700.48163 & 786.43489 & 877.99030 & 975.11633 & 1077.7885 & \\
\hline 10 & 592.59331 & 668.62678 & 750.49845 & 838.12487 & 931.44233 & 1030.4018 & 1134.9651 & 972.06166 & 7 \\
\hline 11 & 721.04936 & 804.24729 & 893.35221 & 988.26926 & 1088.9253 & 1195.2637 & 923.18228 & 826.67993 & 6 \\
\hline 12 & 862.07814 & 952.42108 & 1048.7376 & 1150.9226 & 1258.8943 & 876.76204 & 782.42916 & 693.54140 & 5 \\
\hline 13 & 1015.6789 & 1113.1497 & 1216.6587 & 1326.0912 & 832.66745 & 740.53949 & 653.85026 & 572.60004 & 4 \\
\hline 14 & 1181.8511 & 1286.4345 & 1397.1187 & 790.77458 & 700.87689 & 616.41518 & 537.38923 & 463.79866 & 3 \\
\hline 15 & 1360.5941 & 1472.2765 & 750.96802 & 663.31706 & 581.10160 & 504.32139 & 432.97603 & 367.06499 & 2 \\
\hline 16 & 1551.9077 & 713.13988 & 627.74431 & 547.78488 & 473.26149 & 404.17400 & 340.52222 & 282.30589 & 1 \\
\hline & & 15 & 14 & 13 & 12 & 11 & 10 & 9 & $l \backslash n$ \\
\hline
\end{tabular}


TABLE VI: Critical parameters $\beta=2 m g r_{0}^{2} \hbar^{-2}$ of the Woods-Saxon potential $V(r)=-g /\left[1+\exp \left(r / r_{0}\right)\right]$

\begin{tabular}{|c|c|c|c|c|c|c|c|c|c|}
\hline$n \backslash l$ & 0 & 1 & 2 & 3 & 4 & 5 & 6 & 7 & 8 \\
\hline 1 & 1.7205730 & 8.2135317 & 18.813940 & 33.542490 & 52.406813 & 75.410024 & 102.553558 & 133.83814 & 169.26419 \\
\hline 2 & 9.6742198 & 20.755782 & 35.931282 & 55.231021 & 78.665326 & 106.23821 & 137.9514072 & 173.80576 & 213.80169 \\
\hline 3 & 23.969284 & 39.431300 & 59.073131 & 82.876856 & 110.83541 & 142.94481 & 179.2025315 & 219.60692 & 264.15685 \\
\hline 4 & 44.615717 & 64.332441 & 88.346797 & 116.58590 & 149.01715 & 185.62315 & 226.3936688 & 271.32232 & 320.40492 \\
\hline 5 & 71.614405 & 95.502170 & 123.81377 & 156.42640 & 193.28014 & 234.34169 & 279.5910514 & 329.01551 & 382.60663 \\
\hline 6 & 104.96555 & 132.96409 & 165.51246 & 202.44471 & 243.67452 & 289.15197 & 338.8462258 & 392.73727 & 450.81159 \\
\hline 7 & 144.66921 & 176.73264 & 213.46842 & 254.67366 & 300.23751 & 350.09364 & 404.2000006 & 462.52874 & 525.06071 \\
\hline 8 & 190.72542 & 226.81735 & 267.69958 & 313.13737 & 362.99747 & 417.19781 & 475.6851818 & 538.42365 & 605.38815 \\
\hline 9 & 243.13418 & 283.22488 & 328.218988 & 377.85407 & 431.97649 & 490.48934 & 553.3285074 & 620.44998 & \\
\hline 10 & 301.89551 & 345.96008 & 395.03646 & 448.83786 & 507.19210 & 569.98836 & 637.1520405 & 591.78238 & 7 \\
\hline 11 & 367.00940 & 415.02662 & 468.15961 & 526.09990 & 588.65845 & 655.71141 & 579.47499 & 513.05975 & 6 \\
\hline 12 & 438.47587 & 490.42734 & 547.59441 & 609.64918 & 676.38713 & 568.32987 & 502.26737 & 440.35862 & 5 \\
\hline 13 & 516.29490 & 572.16450 & 633.34572 & 699.49305 & 558.22885 & 492.55183 & 431.02140 & 373.63861 & 4 \\
\hline 14 & 600.46650 & 660.23992 & 725.41749 & 549.06873 & 483.79929 & 422.67311 & 365.69043 & 312.85154 & 3 \\
\hline 15 & 690.99068 & 754.65510 & 540.75901 & 475.91083 & 415.20481 & 358.64093 & 306.21917 & 257.93944 & 2 \\
\hline 16 & 787.86743 & 533.21997 & 468.80008 & 408.52232 & 352.38667 & 300.39309 & 252.54154 & 208.83194 & 1 \\
\hline & & 15 & 14 & 13 & 12 & 11 & 10 & 9 & $l \backslash n$ \\
\hline
\end{tabular}


TABLE VII: Critical parameters $\beta=2 m g r_{0}^{2} \hbar^{-2}$ of the Woods-Saxon potential $V(r)=-g /\left[1+0.001 \exp \left(r / r_{0}\right)\right]$.

\begin{tabular}{|c|c|cccccccc|}
\hline$n \backslash l$ & 0 & 1 & 2 & 3 & 4 & 5 & 6 & 7 & 8 \\
\hline 1 & .050143914 & .21236959 & 45411555 & .77426459 & 1.1724439 & 1.6484948 & 2.2023382 & 2.8339302 & 3.5432443 \\
2 & .40525072 & .74663148 & 1.1653050 & 1.6617334 & 2.2359950 & 2.8880871 & 3.6179882 & 4.4256753 & 5.3111276 \\
3 & 1.0529125 & 1.5665339 & 2.1583382 & 2.8284741 & 3.5768855 & 4.4034793 & 5.3081667 & 6.2908725 & 7.3515353 \\
4 & 1.9863174 & 2.6709161 & 3.4345909 & 4.2772382 & 5.1986738 & 6.1987162 & 7.2772089 & 8.4340224 & 9.6690512 \\
5 & 3.2060640 & 4.0606118 & 4.9951932 & 6.0094520 & 7.1030599 & 8.2757388 & 9.5272608 & 10.857441 & 12.266128 \\
6 & 4.7125157 & 5.7361307 & 6.8408115 & 8.0259370 & 9.2910171 & 10.635665 & 12.059576 & 13.562505 & 15.144255 \\
7 & 6.5057952 & 7.6977523 & 8.9718549 & 10.327215 & 11.763171 & 13.279221 & 14.874975 & 16.550125 & 18.304425 \\
8 & 8.5859457 & 9.9456459 & 11.388595 & 12.913644 & 14.519959 & 16.206914 & 17.974033 & 19.820942 & 21.747345 \\
9 & 10.952985 & 12.479924 & 14.091224 & 15.785486 & 17.561701 & 19.419123 & 21.357183 & 23.375440 & \\
10 & 13.606920 & 15.300668 & 17.079885 & 18.942937 & 20.888645 & 22.916139 & 25.024758 & 20.137676 & 7 \\
11 & 16.547758 & 18.407936 & 20.354690 & 22.386154 & 24.500987 & 26.698194 & 18.543603 & 16.804665 & 6 \\
12 & 19.775499 & 21.801777 & 23.915725 & 26.115262 & 28.398884 & 16.962111 & 15.318555 & 13.753200 & 5 \\
13 & 23.290146 & 25.482228 & 27.763062 & 30.130362 & 15.389823 & 13.842647 & 12.373427 & 10.982209 & 4 \\
14 & 27.091699 & 29.449318 & 31.896761 & 13.822777 & 12.372904 & 11.000819 & 9.7065447 & 8.4901060 & 3 \\
15 & 31.180159 & 33.703074 & 12.256127 & 10.904356 & 9.6302842 & 8.4339165 & 7.3152612 & 6.2743276 & 2 \\
16 & 35.555527 & 10.683731 & 9.4306340 & 8.2552072 & 7.1574531 & 6.1373746 & 5.1949761 & 4.3302634 & 1 \\
\hline
\end{tabular}


FIG. 1: Function $F_{0}(\beta)=\lim _{r \rightarrow \infty} \chi_{0}^{\prime}(r)$ for the Yukawa potential $(l=0)$. Zeros of $F_{0}(\beta)$ present critical parameters $\beta_{n}$.

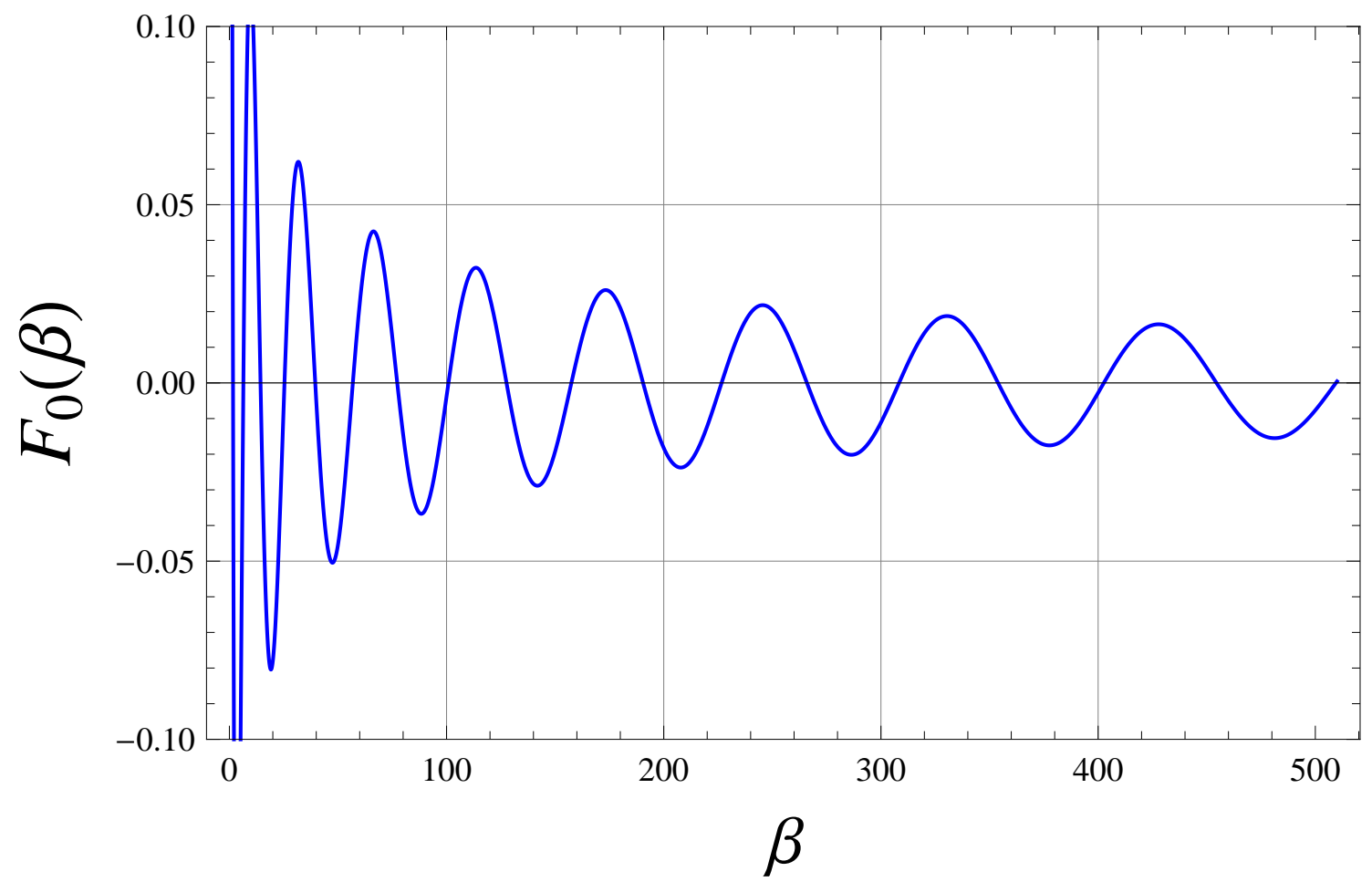


FIG. 2: Function $\tilde{F}_{7}(\beta)=\lim _{r \rightarrow \infty} \eta(r)$ for the Gaussian potential $(l=7)$. The abscissas of the points of intersection of $t=\widetilde{F}_{7}(\beta)$ with lines $t=\delta_{l}-\pi / 2+\pi n$ give the desired critical parameters $\beta=\beta_{n}$.

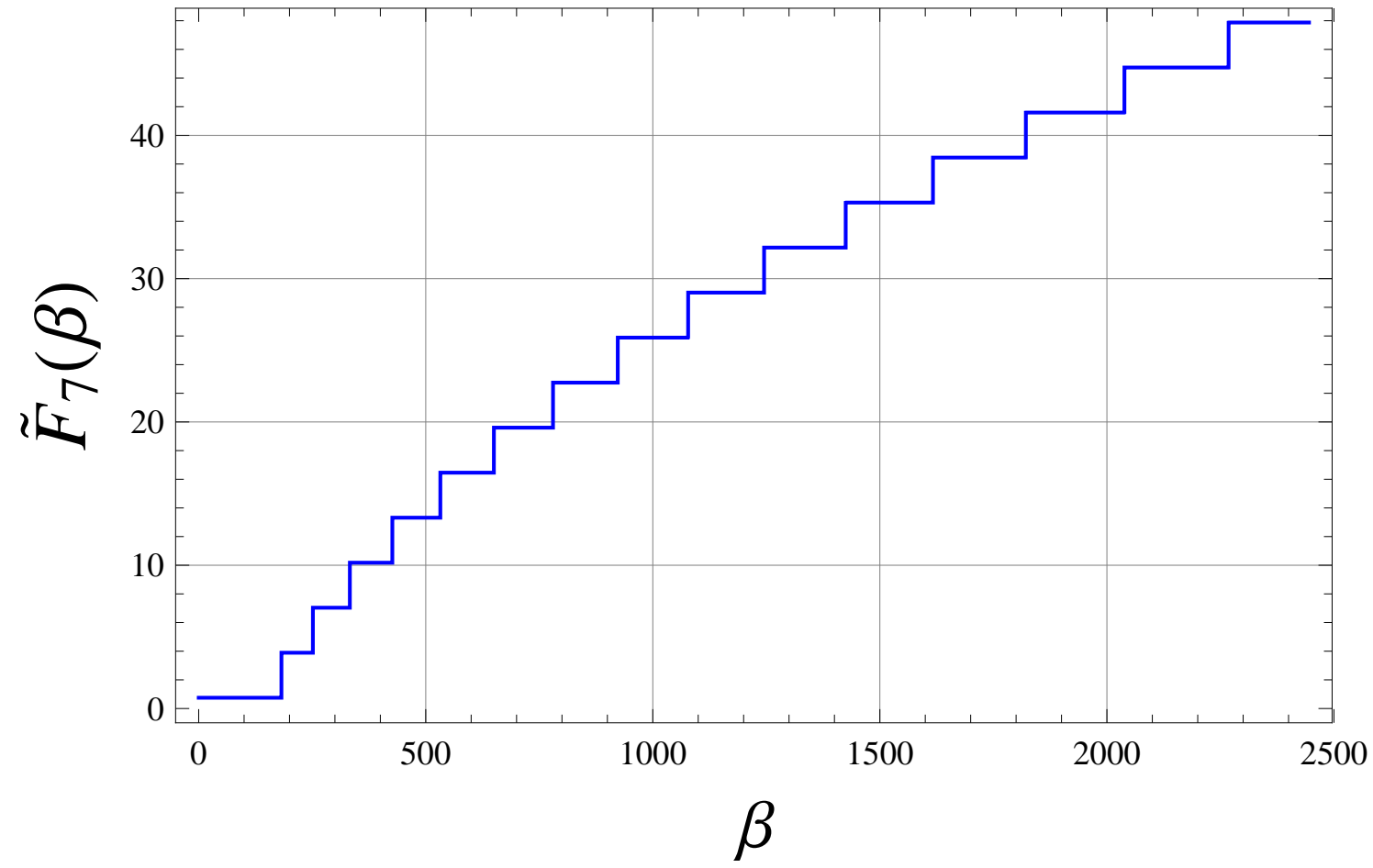

